\title{
BMJ Open Knowledge and perspectives of the new National Cervical Screening Program: a qualitative interview study of North Queensland women -'I could be that one percent'
}

To cite: Nagendiram A, Bidgood R, Banks J, et al. Knowledge and perspectives of the new National Cervical Screening Program: a qualitative interview study of North Queensland women-'I could be that one percent'. BMJ Open 2020;10:e034483. doi:10.1136/ bmjopen-2019-034483

- Prepublication history and additional material for this paper are available online. To view these files, please visit the journal online (http://dx.doi. org/10.1136/bmjopen-2019034483).

Received 24 September 2019 Revised 11 December 2019 Accepted 24 January 2020

Check for updates

(C) Author(s) (or their employer(s)) 2020. Re-use permitted under CC BY-NC. No commercial re-use. See rights and permissions. Published by BMJ.

${ }^{1}$ School of Medicine and Dentistry, James Cook University, Mackay, Queensland, Australia

${ }^{2}$ Canelands Medical Centre, Mackay, Queensland, Australia

Correspondence to Archana Nagendiram; archana.nagendiram@my.jcu. edu.au

\section{ABSTRACT}

Objectives To investigate women's understanding and attitudes towards the National Cervical Screening Program (NCSP) and to explore methods to improve screening participation.

Design Semi-structured face-to-face interviews were conducted through convenience and snowball sampling. Thematic analysis occurred using the interpretivist framework.

Setting A private general practice in North Queensland. Participants Women between the ages of 18 and 74 who attended the general practice were eligible to participate. Fourteen women between 20 and 58 years old were interviewed.

Results Participants were concerned that the new NCSP would miss cancer due to longer screening intervals and reliance on primary human papilloma virus (HPV) testing. They believed that young women are at increased risk of cervical cancer, due to perceived HPV vaccine ineffectiveness and parent objection to vaccination. Most participants were not agreeable to self-sampling and preferred their doctor to perform screening. Personal and practitioner beliefs influenced a woman's screening participation. Personal factors include being healthy for themselves and their family, previous abnormal smears and family history of cancer. Emphasis was placed on feeling 'comfortable' with their practitioner which included patient rapport and gender preference. Proposed methods to improve cervical screening included education programmes, advertising campaigns, general practitioner interventions and improving accessibility.

Conclusions It is apparent that women are hesitant about the new NCSP. However, when provided with additional information they were more amenable to the changes. This highlights the need to improve awareness of cervical screening and the new NCSP.

\section{BACKGROUND}

While the National Cervical Screening Program (NCSP) has more than halved cervical cancer mortality, only $54 \%-56 \%$ of eligible Australian women ${ }^{1}$ participated in screening between January 2016 and June
Strengths and limitations of this study

- Interviews explored participants' attitudes towards the new National Cervical Screening Program, where there is currently little research.

- Individual interviews were conducted until theoretical data saturation and the interview template was piloted.

- Convenience and snowball sampling through a private general practice may have led to inaccurate sample representation.

- Most participants were well-screened for cervical cancer, which may not be inclusive of high-risk groups.

- The study was completed in an outer regional centre and may not apply to the general Australian population.

2017. This is especially concerning as over $90 \%$ of women diagnosed with cervical cancer were under-screened or never-screened. ${ }^{2}$ Women from lower socioeconomic status, rural areas or those that identify as Aboriginal and Torres Strait Islander have lower screening participation rates and higher cervical cancer mortality. ${ }^{1}$ Recently, the NCSP has been changed and has moved away from traditional pap smears, towards a 5 yearly cervical screening test (CST) for human papilloma virus (HPV) with reflex cytology. ${ }^{2}$ Australian women begin cervical screening at 25 years old and have an exit CST from 70 to 74 years old. Women over 30 years who have never been or are currently under-screened are also eligible for self-sampling, consisting of a high vaginal swab for HPV. ${ }^{2}$

As per the WHO guidelines, there has been a global shift to HPV-based cervical screening. ${ }^{4}$ Worldwide, countries including the UK, Ireland, Netherlands and Turkey have implemented national HPV-based 
cervical screening, with many more transitioning towards primary HPV testing. ${ }^{56}$ Previous international studies stated most women are unaware of the use of primary HPV testing ${ }^{7}$ and that some women may experience a negative emotional response towards primary HPV testing as it is a sexually transmitted infection. ${ }^{8}{ }^{9}$ Due to the recent nature of changes to cervical screening, there is limited qualitative literature assessing Australian women's attitudes towards the new NCSP. One Australian study used thematic analysis of an online petition against the new NCSP, revealing that the greatest concerns were missing cancer due to prolonged screening intervals and the delay in screening age. ${ }^{10}$

The researchers' primary aim is to explore North Queensland women's awareness and perceptions of the new NCSP. Knowledge of cervical cancer, factors that affect screening participation and methods to improve screening awareness were also investigated.

\section{METHODS}

Authors followed the Consolidated Criteria for Reporting Qualitative Health Research (COREQ).

\section{Patient selection and setting}

Women between 18 and 74 years old who attended a specific North Queensland general practice were eligible for this study with convenience and snowball sampling. This project was conducted with a concurrent quantitative survey. Receptionists provided waiting room surveys and an expression of interest form for a follow-up interview. Quantitative results collected through waiting room surveys will be published separately. The interviews were advertised on the practice's Facebook page and participants were encouraged to promote the study to their family and friends. Women who expressed interest were contacted by phone or email.

\section{Data collection}

A semi-structured, face-to-face individual interview was conducted at the participant's convenience at their home or a hospital meeting room between March and April 2019. The average length of the interview was $20 \mathrm{~min}$. All interviews were conducted by the primary author, a female medical student (AN) with training in qualitative research. The interviewer (AN) may have some personal bias as she is eligible for cervical screening. No participants were known to $\mathrm{AN}$ and participants were aware that this interview was part of an Honours project.

The piloted interview guide included questions about knowledge of cervical cancer and screening, opinions regarding the NCSP, barriers to screening and thoughts to improve screening participation. During the interview, participants were asked their opinions of the new NCSP, both with their baseline knowledge and after the interviewer provided basic information on the programme. Additionally, the interviewer determined screening status in the interview. Screening status was defined using the previous guidelines due to the current transition to the new NCSP. A 'well-screened' participant had undergone cervical screening in the last 2 years while, an 'underscreened' participant was overdue for screening.

The interview guide (online supplementary appendix 1) was developed after analysis of the existing literature $^{11} 12$ and discussion among the investigators, including a general practitioner and a research officer, both experienced in qualitative research. Prior to the interview, participants signed a consent form for the interview to be audio-recorded. The interviews were transcribed verbatim, including notes on non-verbal cues and were uploaded to NVivo. Transcribed interviews were not returned to participants for comments as there was no ambiguity that required clarification. Further, some methodological literature suggests it may provide little benefit to improving data accuracy and it may lead to misrepresentation of the original data. ${ }^{13}$ No repeat interviews were conducted. Data collection continued until theoretical data saturation was achieved.

\section{Patient and public involvement}

No participants were involved with the development of this study protocol or data analysis.

\section{Data analysis}

Transcribed interviews were analysed using NVivo V.12 ${ }^{14}$ using thematic analysis and the interpretivist paradigm. Data was analysed under relevant headings, for example 'Attitudes towards NCSP' and 'Factors affecting screening participation' but no themes were predetermined. AN completed line-by-line coding to identify the preliminary themes and codes in the data, which were validated by another author (JB) with no significant changes to coding required. Data was further analysed to cluster themes under the appropriate research questions to create a thematic schema.

\section{RESULTS}

\section{Participant characteristics}

Twenty-eight women expressed interest to be interviewed and were contacted by phone or email. Fourteen women consented to be interviewed, with four participants identified by convenience sampling and ten by snowball recruitment. Table 1 displays individual participant characteristics. The age of participants ranged between 20 and 58 years old. Most women participated in screening and only two were considered under-screened as per the previous NCSP.

\section{Themes}

Thematic analysis revealed three major themes on participant's attitudes towards the NCSP: 'fear of missing cancer', 'younger women are at greater risk', 'selfsampling' and 'change is good'. Screening beliefs were subdivided into personal and practitioner factors. Based on participants' suggestions, four potential intervention strategies emerged. Table 2 displays illustrative quotes for 


\begin{tabular}{lll}
\hline Table 1 & Demographic tables & \\
\hline Participant & $\begin{array}{l}\text { Age } \\
\text { (years) }\end{array}$ & Screening status* \\
\hline P1 & 41 & Well-screened \\
\hline P2 & 42 & Under-screened \\
P3 & 43 & Well-screened \\
\hline P4 & 53 & Well-screened \\
\hline P5 & 30 & Well-screened \\
\hline P6 & 55 & Well-screened \\
\hline P7 & 58 & Well-screened \\
\hline P8 & 33 & Well-screened \\
\hline P9 & 20 & Well-screened \\
\hline P10 & 22 & Well-screened \\
\hline P11 & 57 & Well-screened \\
\hline P12 & 36 & Well-screened \\
\hline P13 & 38 & Well-screened \\
\hline P14 & 35 & Under-screened \\
\hline
\end{tabular}

${ }^{*}$ Participants were considered well-screened if they had not participated in screening the last 2 years. Under-screened participants had not been screened in over 2 years or had never been screened.

these themes. A thematic schema depicting conceptual links throughout the themes is displayed in figure 1 .

\section{Knowledge of cervical cancer and NCSP}

Participants identified genetics and infection as the main causes of cervical cancer. Most participants did not specify HPV as the main cause of cervical cancer but when asked, participants were frequently aware of the link between HPV and cervical cancer. Hormones, contraceptive pill, smoking and toxins were listed by participants as causes of cervical cancer. Women considered family history and sexual activity as the main risk factors for cervical cancer. Other risk factors included not participating in regular screening, previous abnormal smears and being unvaccinated. Participants believed abnormal bleeding and pain were symptoms of cervical cancer though, many women were unsure of the symptoms. Bloating, abnormal discharge and pain during sex (dyspareunia) were also reported by some participants as symptoms of cervical cancer.

Participants understood that cervical screening occurs using pap smears and often could describe that pap smears look for abnormal cells. Some participants were aware of the change to screening and could correctly identify that screening now occurs 5-yearly. Women stated that cervical screening should occur in their early twenties and some said that it began with the onset of sexual activity.

\section{Attitudes towards the new NCSP}

Fear of missing cancer

Participants were anxious that 5 years was 'a long time for cancer to grow' (P12) and may lead to more women diagnosed with cancer. More regular screening was identified as having 'a better chance of picking something up'
(P14) as 'things in our body change very quickly' (P1). There were fears that increased screening intervals may miss cancer, as clinicians may not always get a 'clear swab' (P8) and hence more regular screening was preferable. Additionally, participants were apprehensive that primary HPV testing would miss cancer caused by 'things other than HPV' (P9). They were uncomfortable with the CST not testing for abnormal cells as 'I could be that one percent' (P12) with cervical cancer not caused by HPV. Subsequently, some participants preferred cotesting for both HPV and abnormal cells to receive the benefits of both screening methods.

Women believed that 'every 2 years is easy enough to recall but not 5 years' (P12) and may not remember to participate in screening, due to increased screening intervals. Participants were concerned that increased screening intervals will make women more 'complacent' (P12) in their screening behaviour and will delay screening even longer. They proposed that increased intervals required a register to provide appropriate reminders to women. A participant stated that increased intervals may also affect the feasibility of contacting patients, as after 5 years 'not everyone lives at the same address' (P7).

Those with a history of previous abnormal smears thought 5 yearly screening was 'a little bit concerning' (P8). Participants who had family or friends with cervical cancer were concerned by the increased screening interval as 'they didn't pick up hers until it was too late' (P4). One participant negatively viewed delaying screening until a woman is 25 years, as her friend was diagnosed with cervical cancer earlier than this, and 'if they didn't catch it early, she'd be dead' (P2). These personal experiences with abnormal smears or cervical cancer made women more 'paranoid' (P4) about cervical cancer.

\section{Young women are at greater risk}

Participants perceived that commencing screening from 25 years old, placed women at increased risk for cancer due to earlier sexual activity of young women. Participants stated that children are 'having sex a lot younger nowadays' (P13). They highlighted that as young women are sexually active from their early teens, it may be 'over 10 years that kids are sexually active before they have screening' (P3). Participants also believed that delaying cervical screening would prevent opportunistic testing for sexually transmitted infections in young women.

While some women identified that the introduction of the Gardasil vaccination was beneficial in reducing cervical cancer incidence, there was also a perception that 'a lot of parents won't let their kids have that' (P5). This raised concerns as to whether unvaccinated women would be protected under the new NCSP in the future. Additionally, participants feared that 'even though you are vaccinated you can still get diseases' (P7) and believed that delaying screening will lead to cervical cancer being missed in women under 25 years. Some participants thought that patients diagnosed with cancer are becoming 'younger and younger' (P2). Younger women were described to be 
Table 2 Themes and illustrative quotes

\section{Theme}

Attitudes to NCSP

Fear of missing cancer

A woman's body So obviously, it's important to try and catch it early and a couple of years can make a huge changes quickly difference. So, I think it probably would have been better if they left it the way that it was. (P10) But like stretching it out that bit further, it worries me that is something going to happen and by the time they come around to screening again, is it going to be too late for me? (P4)

Missing non-HPV cancer If we miss that $1 \%$, what's that out of 100 ? That's still one person. One person out of every hundred we're missing, that's sad. That's someone's mother, someone's daughter. (P12) I would think put some women at risk, that are not exposed to HPV and would get it otherwise. (P2)

Women will forget $\quad$ And then it will get to that 5 years and people will still go, 'Well l'll just wait another 6 months.' (P5) I think there will be too many people that get complacent and I think because of it too many people will get sick. (P12)

Personal experiences Considering that l've had smears that have been not... a little bit concerning, l'd be concerned about that. (P8)

A friend of mine got cervical cancer when she was $21 \ldots$ If they didn't catch it early, she'd be dead. (P2)

Younger women are at greater risk

Earlier sexual activity A lot of children are starting to have sex at 13,14 . At 25 , for some kids that's over 10 years that kids are sexually active before they have screening. (P3)

Vaccine effectiveness Depends on how good the vaccination is and the stats around how effective that is. Because we know that vaccinations aren't $100 \%$. (P8)

Maybe that's what contributed to the 25 age testing that a lot of younger women have had the Gardasil vaccine but even then, a lot of parents won't let their kids have that. (P5)

More young people have And I've heard that there has been cases of younger ones getting cervical cancer. Stuff that cancer normally you don't see til you are older and now coming up in younger women. (P4) I think younger people are at higher risk. (P2)

The changes are good

Trust in medicine I think l've got faith in the doctors because there must be a reason as to why they are doing that. (P11)

Reduced discomfort Well I like the idea of not having to go back every 2 years and not be in that uncomfortable position. (P14)

Earlier detection of I guess it starts somewhere doesn't it. If they pick up one thing, they can prevent it from leading cancer to something else. (P13)

Screening beliefs

Personal

To be healthy Just because I want to be healthy, like I go to the dentist and have a normal check-up every year. This is part of the routine. (P11)

To be there for family It wouldn't just affect me, it would affect my whole family. I couldn't be selfish like that, to not get it done for whatever reason. Because, once you're gone... you've left and not destroyed but you've upset your whole family by not going to look after yourself when it's just a simple test. (P4)

Hx cancer/abnormal My grandma got really sick with cancer and so it kind of has impacted me to make sure I try and smears look after myself. (P10)

A long time ago I did have an abnormal smear. So, I guess knowing that, not wanting to go through that again making sure I'm keeping on top of that, so it doesn't happen again. (P13)

Practitioner

Feeling comfortable As long as I felt comfortable with the doctor. Their bedside manner was nice, they were clean, the instruments they used was sterile, they followed all their proper PPE precautions. (P12)

Trust in regular GP I always just go to my GP. (P9)

.... because I had been going to him for a long time. (P4)

Continued 
Table 2 Continued

Theme

Male practitioner

I just feel more comfortable with a female because I'm female (P11)

Potential interventions

Education programme

General practitioner

Media campaign

at 'higher risk' (P2) of cervical cancer and participants felt that earlier detection of cervical cancer in women would improve their survival rates.

\section{Change is good}

Several participants expressed their 'faith in the doctors' (P11) and believed that screening would not have changed without underlying improvements in science. They perceived that 'testing is that good' (P7) if screening intervals have increased from every 2 years to 5 years. Participants highlighted that HPV testing is a positive change as it would 'catch it (HPV)' (P11) before abnormal cells became detectable by a traditional pap smear. They accepted that if HPV was 'linked so strongly'
(P9) with cervical cancer, primary HPV testing was a beneficial change to cervical screening. After participants were provided information on the new NCSP and cervical cancer's slow progression, they were more receptive towards the new guidelines. An additional benefit of the new NCSP was that reduced screening would 'make life easier' (P6) for women, as cervical screening was described as 'uncomfortable' (P14) by participants.

\section{Self-sampling}

Participants were generally hesitant to accept selfsampling as they did not 'feel confident enough in myself' (P14) to administer the test. This stemmed from the fear of incorrectly completing the test as they may not 'swab

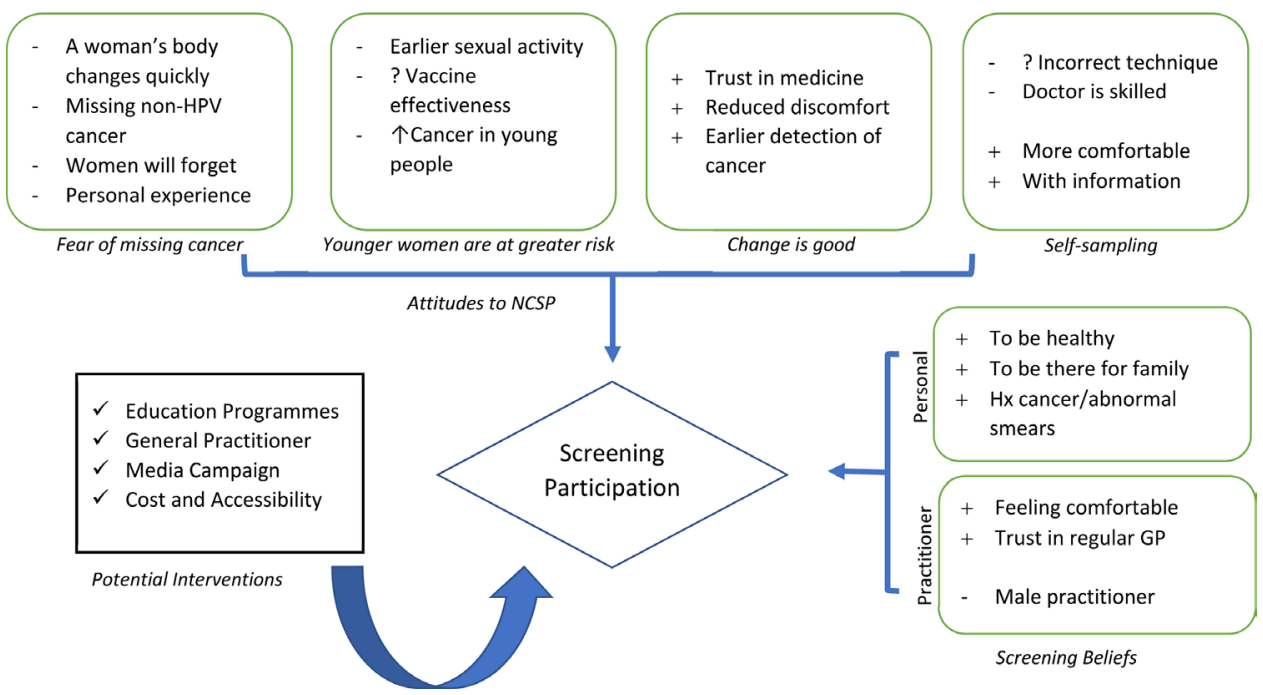

Figure 1 Relationship between identified themes. 
far enough' (P5) or that 'it might be contaminated' (P4). Doctors were thought to be better equipped to perform cervical screening as they had appropriate training to ensure it was 'done properly' (P8). It was perceived that doctors had better visualisation of the cervix as they used a speculum and would take a sample from 'the right places' (P3).

Nonetheless, participants acknowledged that selfsampling would provide women 'more privacy' (P10) and could improve screening participation. The test was deemed to be 'less invasive' (P5) than traditional cervical screening, especially for those who have had previous negative experiences with cervical screening. Participants often had the misconception that self-sampling required a cervical swab. When informed it was in fact, a high-vaginal swab more participants reported they would consider selfsampling. Some participants indicated that they would complete self-sampling if they were provided instructions or shown by a practitioner. Women highlighted that technological improvements allowed for 'smaller traces' (P2) of HPV to be detected and this made self-sampling more acceptable.

\section{Factors affecting screening participation Personal}

Participants explained that cervical screening was a part of their normal health 'routine' (P11). Screening allowed them to have 'peace of mind' that they were healthy, and it allowed them to receive 'further help' (P1) if required. Many women emphasised that their family was the key reason they had cervical screening. They described their sense of responsibility to their family who required them to 'make the right choices' (P3) by undergoing screening. Participants disclosed that cancer could destroy their families and thus they chose to screen. A woman's personal experiences with cancer shaped her willingness to participate in cervical screening. A history of abnormal smears motivated women to 'keep on top' (P13) of their screening to prevent reoccurrence. Women with a family history of cancer had firsthand experience as to how it devastated their families, which motivated them to undergo regular cervical screening.

\section{Practitioner}

Several participants described feeling 'comfortable' (P10) with their general practitioner as a facilitator to screening participation. This feeling of being 'comfortable' was influenced by the doctor's bedside manner, professional behaviour and hygienic technique throughout the procedure. Some women expressed that they trusted their regular doctor due to their pre-existing relationship, as they have known them for a 'long time' (P4). Participants frequently mentioned their practitioner's gender when discussing screening participation. Most participants indicated that they are more 'comfortable' with a female doctor as she 'has the same bits' (P2). However, some women had no gender preference as cervical screening is 'just something that happens' $(\mathrm{P} 7)$ and one participant thought that 'once you have a few kids, you're over it' (P4).

\section{Improving CST awareness and participation \\ Cost and accessibility}

A lack of bulk-billed services in regional areas was identified by women as a key barrier to cervical screening participation. The cost of cervical screening was a 'deterrent' (P5) for attending the general practitioner for screening, especially in low socioeconomic women as 'their priority is probably not money for them, it's going to be feeding their children' (P5). Participants also emphasised that cervical screening is not bulk-billed, unlike breast and bowel cancer. Women expressed the need for increased accessibility of cervical screening, especially in regional communities. It was suggested that cervical screening should have 'pop-up' (P9) clinics, as seen with blood donation and breast screening.

\section{Media campaigns}

The role of a media campaign to potentially increase cervical screening awareness was discussed by participants. Participants proposed that television advertisements should be aired to encourage discussion about screening. Social media was deemed to be a valuable platform to educate women about cervical screening due to its 'really big role in young women's lives' (P9) and the interaction remained 'private' (P2). Women also suggested that an ambassador should lead the discussion on cervical cancer, such as in England when a celebrity 'found out on Big Brother that she had cervical cancer' (P3). Participants expressed that it is important to hear personal stories from survivors as it provides a 'scare factor' (P8) and would encourage screening.

\section{Education programs}

Participants explained the importance of education to improve screening participation, especially though sex education in schools to ensure young women have the 'right information' (P5) about HPV and cervical cancer. Education programmes should inform women about the purpose of the Gardasil vaccine, the NCSP and symptoms of cervical cancer. Participants believed that education will reduce any fears related to screening and solidify 'how important it is' (P12). It was also suggested that education sessions should also occur at universities, playgroups and the workplace.

\section{General practitioner}

Participants emphasised the importance of their general practitioner as a trusted source of information about cervical screening as 'you listen to them' (P6). It was indicated that general practitioners should facilitate opportunistic discussions about screening, especially when a woman turned 25 years old. Practices should display information in the waiting rooms and provide written information such as pamphlets or fact sheets, with this information being 'very direct, black and white' (P1) to increase the likelihood that a woman will read it. It was 
also proposed that general practices should provide information by phone or email.

\section{DISCUSSION}

It became apparent that women were hesitant about the new NCSP, as they were apprehensive that screening will miss cancers due to primary HPV testing and increased screening intervals. A Canadian study by Ogilvie $e t$ al stated that primary HPV testing was acceptable, though acceptability decreased when combined with increased screening intervals. ${ }^{15}$ Participants' objections towards the change in screening may be due to the general public's fatalistic view of cancer and belief that frequent testing leads to earlier diagnosis. ${ }^{16}$ A qualitative Irish study highlighted that even when women understood HPV causes cancer, they were anxious about screening changes due to their attachment to traditional pap smears. ${ }^{17}$ Participants were afraid that the new programme would endanger young women as they were perceived to be at increased risk of cervical cancer. This was due to participant concerns that young women are becoming sexually active earlier and queries over the effectiveness of the Gardasil vaccine. In reality however, women over 50 years are at higher risk for cervical cancer and this misconception of age-related risk may indicate the need for more patient education. ${ }^{18}$ Moreover, the new screening programme is predicted to have a greater impact in cervical cancer mortality in unvaccinated cohorts $(36 \%)$ over vaccinated cohorts $(29 \%) .{ }^{19}$

It is noteworthy that participants became more accepting of primary HPV screening after they were provided additional information explaining the new NCSP. This is congruent with Waller $e t a t^{20}$ who described that women's anxieties surrounding HPV reduced as health literacy increased. Participants suggested a variety of methods to improve screening including education programmes concentrating on young women. Minimal education occurs about cervical cancer and HPV in schools. Parents may pereceive it is not their responsibility to educate their children on this topic, though a school-based education programme was an acceptable solution to this. ${ }^{21}$ Media and social media campaigns were proposed to increase awareness. Participants suggested the use of cancer survivors as ambassadors, though past research has suggested an emphasis on information from organisations over personal anecdotes. ${ }^{22}$ The general practitioner is a trusted and valued source of information and should provide interventional health education with eligible women. ${ }^{23}{ }^{24} \mathrm{~A}$ systematic literature review explains that patient's likelihood to participate in preventive screening is linked directly to the quality of discussion between clinician and patient, with an emphasis on shared decision making rather than a simple recommendation. ${ }^{25}$ More research should occur in general practice to develop and evaluate interventions in an Australian context in order to improve cervical screening.
Finally, logistical concerns prevented women from participating in screening. Previous literature demonstrates that reducing economic and geographic barriers improve disadvantaged women's likelihood to participate in cervical screening. ${ }^{26}$ Unlike other government mandated screening programmes, such as bowel and breast screening, women bear the burden of cost for cervical screening when attending their appointment with their general practitioner. Government policymakers should reassess the costs involved in cervical screening and consider expanding the availability of bulk-billed services.

Screening participation is influenced by personal and practitioner factors. A woman's personal factors include: to be healthy, to be there for family, history of abnormal smears or cancer. A woman's comfort in her relationship with her practitioner influenced her screening behaviour. This was determined by trust in her regular doctor, gender preference and a general feeling of comfort. A previous qualitative literature review similarly discussed that screening provides reassurance of health. Though unlike this study, it also iterated that the emotional response elicited by the test and previous negative experiences hindered participation. ${ }^{27}$ This may be as the literature review encompassed studies that included under-screened populations, while this study only included two underscreened women.

This study provides timely qualitative research on participant's perceptions of the new NCSP and will provide valuable data on these changes. Researchers conducted interviews using a piloted interview template until theoretical data saturation. It was also conducted in parallel to a quantitative study as an explanatory component and allowed for data triangulation. However, sampling at one private regional general practice may have led to a skewed representation of the population and may not include high-risk groups for cervical cancer. In addition, snowball sampling may have led to women with more positive health-seeking behaviours to participate in this project. The use of the interpretivist paradigm may allow for the impact of the primary researcher bias (AN) though, this was minimised by a second author (JB) validating thematic analysis.

\section{CONCLUSION}

Women are apprehensive about the new changes to the NCSP, as they believe that increased screening intervals and primary HPV testing may lead to missed cervical cancers. Personal and practitioner factors affect screening participation, with emphasis on maintaining good health and feeling 'comfortable' with their doctor. Cervical cancer participation could be improved by increasing education about cervical cancer and the new NCSP through schools, advertising campaigns and the general practitioner. It was also emphasised that the cost and accessibility of screening should be revised. 
Acknowledgements The authors would like to thank the staff of the general practice where this study was conducted for their assistance with participant recruitment. We also would like to acknowledge the women who participated in interviews for their time and opinions. Thank you to all those who collaborated on this paper and for those who have taken the time to read it.

Contributors AN designed the study, conducted interviews, transcribed interviews, coded data, conducted thematic analysis and was primary author of the manuscript. RB assisted with logistical considerations for data collection at the general practice. JB assisted with study design, designing the interview template, reviewed thematic analysis and provided intellectual input into the manuscripts. $\mathrm{CH}$ assisted with study design, creating the interview template and provided intellectual input into the manuscripts.

Funding This work was supported by the College of Medicine and Dentistry, James Cook University (grant number JCU-QLD-792241).

Competing interests None declared.

Patient consent for publication Not required.

Ethics approval Ethical approval was provided by the James Cook University Human Research Ethics Committee (H7465). Participants completed a consent form that stated that they agreed to a recorded interview.

Provenance and peer review Not commissioned; externally peer reviewed.

Data availability statement Data are available upon reasonable request.

Open access This is an open access article distributed in accordance with the Creative Commons Attribution Non Commercial (CC BY-NC 4.0) license, which permits others to distribute, remix, adapt, build upon this work non-commercially, and license their derivative works on different terms, provided the original work is properly cited, appropriate credit is given, any changes made indicated, and the use is non-commercial. See: http://creativecommons.org/licenses/by-nc/4.0/.

ORCID iDs

Archana Nagendiram http://orcid.org/0000-0002-9210-2252

Jennifer Banks http://orcid.org/0000-0003-1520-7488

Clare Heal http://orcid.org/0000-0002-5041-2666

\section{REFERENCES}

1 AlHW. Cervical screening in Australia 2019, 2019. Available: https:// www.aihw.gov.au/reports/cancer-screening/cervical-screening-inaustralia-2019/contents/table-of-contents [Accessed 12 Sep 2019].

2 MSAC. National cervical screening program renewal: Executive summary, 2016. Available: http://www.health.gov.au/internet/msac/ publishing.nsf/Content/1276-public [Accessed 12 Sep2019].

3 Arbyn M, Verdoodt F, Snijders PJF, et al. Accuracy of human papillomavirus testing on self-collected versus clinician-collected samples: a meta-analysis. Lancet Oncol 2014;15:172-83.

4 WHO. Comprehensive cervical cancer prevention and control - a healthier future for girls and women, 2013. Available: https://www. who.int/reproductivehealth/publications/cancers/9789241505147/en/ [Accessed 22 Nov 2019].

5 Maver PJ, Poljak M. Primary HPV-based cervical cancer screening in Europe: implementation status, challenges, and future plans. Clin Microbiol Infect 2019. doi:10.1016/j.cmi.2019.09.006. [Epub ahead of print: 17 Sep 2019].

6 Chrysostomou AC, Stylianou DC, Constantinidou A, et al. Cervical cancer screening programs in Europe: the transition towards HPV vaccination and population-based HPV testing. Viruses 2018;10. doi:10.3390/v10120729. [Epub ahead of print: 19 Dec 2018].

7 Patel H, Moss EL, Sherman SM. Hpv primary cervical screening in England: women's awareness and attitudes. Psychooncology 2018;27:1559-64.
8 McCaffery K, Waller J, Nazroo J, et al. Social and psychological impact of HPV testing in cervical screening: a qualitative study. Sex Transm Infect 2006;82:169-74.

9 Daley EM, Perrin KMK, McDermott RJ, et al. The psychosocial burden of HPV: a mixed-method study of knowledge, attitudes and behaviors among HPV+ women. J Health Psychol 2010;15:279-90.

10 Dodd $\mathrm{RH}$, Obermair HM, McCaffery KJ. A thematic analysis of attitudes toward changes to cervical screening in Australia. JMIR Cancer 2019;5:e12307.

11 Kwok C, White K, Roydhouse JK. Chinese-Australian women's knowledge, facilitators and barriers related to cervical cancer screening: a qualitative study. J Immigr Minor Health 2011;13:1076-83.

12 Anaman-Torgbor JA, King J, Correa-Velez I. Barriers and facilitators of cervical cancer screening practices among African immigrant women living in Brisbane, Australia. Eur J Oncol Nurs 2017;31:22-9.

13 Hagens V, Dobrow MJ, Chafe R. Interviewee transcript review: assessing the impact on qualitative research. BMC Med Res Methodol 2009;9:47.

14 NVivo for Windows. Version 12 Plus. Melbourne, Vic: QSR International Pty Ltd. 2019.

15 Ogilvie GS, Smith LW, van Niekerk DJ, et al. Women's intentions to receive cervical cancer screening with primary human papillomavirus testing. Int J Cancer 2013;133:2934-43.

16 Obermair HM, Dodd RH, Bonner C, et al. 'It has saved thousands of lives, so why change it?' content analysis of objections to cervical screening programme changes in Australia. BMJ Open 2018;8:e019171.

17 McRae J, Martin C, O'Leary J, et al. "If you can't treat HPV, why test for it?" Women's attitudes to the changing face of cervical cancer prevention: a focus group study. BMC Womens Health 2014;14:64.

18 Elit L. Role of cervical screening in older women. Maturitas 2014;79:413-20.

19 Cancer Council Australia. Medical services Advisory Committee recommendations for HPV testingNational cervical screening program: guidelines for the management of screen-detected abnormalities, screening in specific populations and investigation of abnormal vaginal bleeding, 2017. Available: https://wiki.cancer org.au/australia/Guidelines:Cervical_cancer/Screening/Medical Services_Advisory_Committee_recommendations_for_HPV_testing [Accessed 12 Sep 2019].

20 Waller J, McCaffery K, Kitchener H, et al. Women's experiences of repeated HPV testing in the context of cervical cancer screening: a qualitative study. Psychooncology 2007;16:196-204.

21 Cooper Robbins SC, Bernard D, McCaffery K, et al. "Is cancer contagious?": Australian adolescent girls and their parents: making the most of limited information about HPV and HPV vaccination. Vaccine 2010;28:3398-408.

22 Zhang J, Le G, Larochelle D, et al. Facts or stories? how to use social media for cervical cancer prevention: a multi-method study of the effects of sender type and content type on increased message sharing. Prev Med 2019;126:105751.

23 Jayasinghe Y, Rangiah C, Gorelik A, et al. Primary HPV DNA based cervical cancer screening at 25 years: views of young Australian women aged 16-28 years. J Clin Virol 2016;76 Suppl 1:S74-80.

24 Pitts MK, Dyson SJ, Rosenthal DA, et al. Knowledge and awareness of human papillomavirus (HPV): attitudes towards HPV vaccination among a representative sample of women in Victoria, Australia. Sex Health 2007;4:177-80.

25 Peterson EB, Ostroff JS, DuHamel KN, et al. Impact of providerpatient communication on cancer screening adherence: a systematic review. Prev Med 2016;93:96-105.

26 Spadea T, Bellini S, Kunst A, et al. The impact of interventions to improve attendance in female cancer screening among lower socioeconomic groups: a review. Prev Med 2010;50:159-64.

27 Chorley AJ, Marlow LAV, Forster AS, et al. Experiences of cervical screening and barriers to participation in the context of an organised programme: a systematic review and thematic synthesis. Psychooncology 2017;26:161-72. 